\title{
JOINT HÖLDER CONTINUITY OF PARABOLIC ANDERSON MODEL
}

\author{
YAOZHONG HU AND KHOA LÊ
} ABstract. We show that the random field solution to the parabolic Anderson equation $\left(\partial_{t}-\frac{1}{2} \Delta\right) u=u \diamond \dot{W}$
is jointly Hölder continuous in space and time.

\section{INTRODUCTION}

In this paper we study the Hölder continuity and joint Hölder continuity of the solution to the following multiplicative stochastic heat equation:

$$
\frac{\partial u(t, x)}{\partial t}=\frac{1}{2} \Delta u(t, x)+u(t, x) \dot{W}(t, x),
$$

where the unknown $u=\left\{u(t, x), t \geq 0, x \in \mathbb{R}^{d}\right\}$ is a random field, $\Delta=\sum_{i=1}^{d} \frac{\partial^{2}}{\partial x_{i}^{2}}$ is the Laplacian, and $\dot{W}$ is general mean zero Gaussian noise whose covariance is given by

$$
\mathbb{E}\left(\dot{W}(s, x) \dot{W}(t, y)=\gamma_{0}(t-s) \gamma(x-y), \quad \forall s, t \geq 0, x, y \in \mathbb{R}^{d} .\right.
$$

Here we assume that $\gamma_{0}$ is a locally integrable function and $\gamma(x)=\int_{\mathbb{R}^{d}} e^{\iota x \cdot \xi} \mu(\xi) d \xi$ for some non-negative spectral density $\mu$ which will be specified in the next section. The multiplication between $u$ and $\dot{W}$ is the Wick product, whose precise meaning is given in the following section by using the Skorohod integral.

There are several papers on the Hölder continuity of the solution of stochastic partial differential equation. When the space dimension is 1 and when the noise $\dot{W}$ is space time white it is well-known that the solution is Hölder continuous of exponent $\beta / 2$ in time variable and $\beta$ in space variable $x$ for any $\beta<1 / 2$ (see [2] and [14] for some work and for references).

When the noise is a general Gaussian noise, there are also some work on Hölder continuity in [7], [9], and [10]. However, the Hölder continuity obtained in those papers are not sharp: when the noise is reduced to the one dimensional space-time white noise, one cannot obtain the known Hölder exponents. This paper contains three main contributions. First, we can allow the Gaussian noise $\dot{W}$ to be quite general, including the noise which is rough in space studied in recent work [7], [8], and [9]. Secondly, our results are sharp, they yield optimal Hölder exponents when the noise is reduced to one dimensional space time white noise. Third, we obtain joint Hölder continuity in the sense of [5].

After the completion of the current paper, we have learnt that Balan et.al. in [1] have obtained results along the same line. Although the assumptions on the spatial covariance of the noise are different, the method of [1] also relies on Wiener chaos expansion. However, the current paper proceed further by considering joint Hölder continuity.

\section{Preliminary and notations}

Let us start by introducing our basic notation on Fourier transforms of functions. The space of Schwartz functions on $\mathbb{R}^{d}$ is denoted by $\mathcal{S}=\mathcal{S}\left(\mathbb{R}^{d}\right)$. Its dual, the space of tempered distributions, is $\mathcal{S}^{\prime}$. The Fourier transform of a function $u \in \mathcal{S}$ or distribution $u \in \mathcal{S}^{\prime}$ is defined with the normalization

$$
\mathcal{F} u(\xi)=\int_{\mathbb{R}^{d}} e^{-\iota \xi \cdot x} u(x) d x
$$

so that the inverse Fourier transform is given by $\mathcal{F}^{-1} u(\xi)=(2 \pi)^{-1} \mathcal{F} u(-\xi)$.

2010 Mathematics Subject Classification. Primary 60H15; Secondary 35R60, 60G60.

Key words and phrases. Gaussian process; stochastic heat equation; parabolic Anderson model; multiplicative noise.

$\mathrm{Y}$. Hu is supported by a startup fund from University of Alberta at Edmonton. 
Let $\gamma_{0}(s), 0<s<\infty$ be a real valued positive locally integrable function such that $\gamma_{0}(s-t)$ is a positive definite function of $s, t \in(0, \infty)$. Let $\mu(\xi)$ be a non-negative function on $\mathbb{R}^{d}$.

Let $\mathcal{D}\left((0, \infty) \times \mathbb{R}^{d}\right)$ denote the space of real-valued infinitely differentiable functions with compact support on $(0, \infty) \times \mathbb{R}^{d}$ and let $\left\{W(\varphi), \varphi \in \mathcal{D}\left((0, \infty) \times \mathbb{R}^{d}\right)\right\}$ be a zero-mean Gaussian family defined on a complete probability space $(\Omega, \mathcal{F}, \mathbf{P})$, whose covariance structure is given by

$$
\mathbb{E}[W(\varphi) W(\psi)]=\int_{\mathbb{R}_{+}^{2} \times \mathbb{R}^{d}} \mathcal{F} \varphi(s, \xi) \overline{\mathcal{F} \psi(t, \xi)} \gamma_{0}(s-t) d s d t \mu(\xi) d \xi
$$

where the Fourier transforms $\mathcal{F} \varphi, \mathcal{F} \psi$ are understood as Fourier transforms in spatial variables only.

We denote by $\mathfrak{H}$ the Hilbert space obtained by completion of $\mathcal{D}((0, \infty) \times \mathbb{R})$ with respect to the inner product

$$
\langle\varphi, \psi\rangle_{\mathfrak{H}}=\int_{\mathbb{R}_{+}^{2} \times \mathbb{R}^{d}} \mathcal{F} \varphi(s, \xi) \overline{\mathcal{F} \psi(t, \xi)} \gamma_{0}(s-t) d s d t \mu(\xi) d \xi
$$

We shall use Malliavin calculus to define the stochastic integral ([4] and [13]). If a random variables $F$ is of the smooth and cylindrical form

$$
F=f\left(W\left(\phi_{1}\right), \ldots, W\left(\phi_{n}\right)\right),
$$

with $\phi_{i} \in \mathfrak{H}, f \in C_{p}^{\infty}\left(\mathbb{R}^{n}\right)$ ( $f$ is smooth and $f$ itself and all its partial derivatives have polynomial growth) then its Malliavin derivative is the $\mathfrak{H}$-valued random variable defined by

$$
D F=\sum_{j=1}^{n} \frac{\partial f}{\partial x_{j}}\left(W\left(\phi_{1}\right), \ldots, W\left(\phi_{n}\right)\right) \phi_{j} .
$$

The operator $D$ is closable from $L^{2}(\Omega)$ into $L^{2}(\Omega ; \mathfrak{H})$ and we define the Sobolev space $\mathbb{D}^{1,2}$ as the closure of the space of smooth and cylindrical random variables under the norm

$$
\|F\|_{1,2}=\sqrt{\mathbb{E}\left[F^{2}\right]+\mathbb{E}\left[\|D F\|_{\mathfrak{H}}^{2}\right]} .
$$

If $u \in L^{2}(\Omega ; \mathfrak{H})$, we say that $u$ is in the domain of the divergence operator $\delta$ if there is a square integrable random variable, denoted by $\delta(u)$, such that

$$
\mathbb{E}[\delta(u) F]=\mathbb{E}\left[\langle D F, u\rangle_{\mathfrak{H}}\right] \quad \text { for any } F \in \mathbb{D}^{1,2} .
$$

$\delta(u)$ is also called the Skorohod integral of $u$ and we also denote this integral by $\delta(u)=\int_{\mathbb{R}_{+} \times \mathbb{R}^{d}} u(t, x) W(d t, d x)$.

For any integer $n \geq 0$ we denote by $\mathbf{H}_{n}$ the $n$-th Wiener chaos of $W$. We recall that $\mathbf{H}_{0}$ is simply $\mathbb{R}$ and for $n \geq 1, \mathbf{H}_{n}$ is the closed linear subspace of $L^{2}(\Omega)$ generated by the random variables $\left\{H_{n}(W(\phi)), \phi \in\right.$ $\left.\mathfrak{H},\|\phi\|_{\mathfrak{H}}=1\right\}$, where $H_{n}(x)=e^{\frac{x^{2}}{2}} \frac{d^{n}}{d x^{n}} e^{-\frac{x^{2}}{2}}$ is the $n$-th Hermite polynomial. For any $n \geq 1$, we denote by $\mathfrak{H}^{\otimes n}$ (resp. $\mathfrak{H}^{\odot n}$ ) the $n$-th tensor product (respectively the $n$-th symmetric tensor product) of $\mathfrak{H}$. Then, the mapping $I_{n}\left(\phi^{\otimes n}\right)=H_{n}(W(\phi))$ can be extended to a linear isometry between $\mathfrak{H}^{\odot n}$ (equipped with the modified norm $\left.\sqrt{n !}\|\cdot\|_{\mathfrak{H}} \otimes n\right)$ and $\mathbf{H}_{n}$. The chaos expansion theorem says that any square integrable nonlinear functional $F$ of $W$ can be expressed a

$$
F=\mathbb{E}[F]+\sum_{n=1}^{\infty} I_{n}\left(f_{n}\right)
$$

where the series converges in $L^{2}(\Omega)$, and the elements $f_{n} \in \mathfrak{H}^{\odot n}, n \geq 1$, are determined by $F$.

The Skorohod integral (or divergence) of a random field $u$ can be computed by using the Wiener chaos expansion. More precisely, suppose that $u=\left\{u(t, x),(t, x) \in \mathbb{R}_{+} \times \mathbb{R}\right\}$ is a random field such that for each $(t, x), u(t, x)$ is a square-integrable random variable, then for each $(t, x)$ we have a Wiener chaos expansion of the form

$$
u(t, x)=\mathbb{E}[u(t, x)]+\sum_{n=1}^{\infty} I_{n}\left(f_{n}(\cdot, t, x)\right) .
$$

Then $u$ belongs to the domain of the divergence operator (that is, $u$ is Skorohod integrable with respect to $W)$ if and only if the following series converges in $L^{2}(\Omega)$

$$
\delta(u)=\int_{0}^{\infty} \int_{\mathbb{R}^{d}} u(t, x) W(d t, d x)=W(\mathbb{E}[u])+\sum_{n=1}^{\infty} I_{n+1}\left(\widetilde{f}_{n}\right),
$$


where $\widetilde{f}_{n}$ denotes the symmetrization of $f_{n}$ in all its $n+1$ variables.

We shall use the following lemma which has been used in [7], [8], [9].

Lemma 2.1. For $m \geq 1$ let $\alpha \in(-1,1)^{m}$ and set $|\alpha|=\sum_{i=1}^{m} \alpha_{i}$. For $t \in[0, T]$, the $m$-th dimensional simplex over $[0, t]$ is denoted by $T_{m}(t)=\left\{\left(r_{1}, r_{2}, \ldots, r_{m}\right) \in \mathbb{R}^{m}: 0<r_{1}<\cdots<r_{m}<t\right\}$. Then there is a constant $c>0$ such that

$$
J_{m}(t, \alpha):=\int_{T_{m}(t)} \prod_{i=1}^{m}\left(r_{i}-r_{i-1}\right)^{\alpha_{i}} d r \leq \frac{c^{m} t^{|\alpha|+m}}{\Gamma(|\alpha|+m+1)},
$$

where by convention, $r_{0}=0$.

We shall also use the following classical result. The Mittag-Leffler function is defined as

$$
E_{\alpha}(z)=\sum_{n=0}^{\infty} \frac{z^{n}}{\Gamma(\alpha n+1)} .
$$

It is well-known that $\left|E_{\alpha}(z)\right| \leq C e^{|z|^{\frac{1}{\alpha}}}$. By Stirling formula one also have

$$
\sum_{n=1}^{\infty} \frac{|z|^{n}}{(n !)^{\alpha}} \leq C \exp \left\{|z|^{\frac{1}{\alpha}}\right\}
$$

We shall use $c$ and $C$ to denote constants. For example, we may write $c^{2 n}$ as $c^{n}$.

\section{Existence and uniqueness Via chaos expansions}

Let $u=\left\{u(t, x), 0 \leq t \leq T, x \in \mathbb{R}^{d}\right\}$ be a real-valued stochastic process such that for all $t \in[0, T]$ and $x \in \mathbb{R}^{d}$ the process $\left\{p_{t-s}(x-y) u(s, y) \mathbf{1}_{[0, t]}(s), 0 \leq s \leq t, y \in \mathbb{R}^{d}\right\}$ is Skorohod integrable, where $p_{t}(x)=(2 \pi t)^{-d / 2} \exp \left(-\frac{|x|^{2}}{2}\right)$ is the heat kernel on the real line related to $\frac{1}{2} \Delta$.

Definition 3.1. We say that $u$ is a mild solution of (1.1) if for all $t \in[0, T]$ and $x \in \mathbb{R}^{d}$ we have

$$
u(t, x)=p_{t} * u_{0}(x)+\int_{0}^{t} \int_{\mathbb{R}^{d}} p_{t-s}(x-y) u(s, y) W(d s, d y) \quad \text { a.s. },
$$

where the stochastic integral is understood in the Skorohod sense.

We make the following assumptions on the covariance structure of the noise $\dot{W}$.

Hypothesis 3.2. There are positive constants $\alpha_{0} \in[0,1]$ and $C$ such that

$$
\gamma_{0}(t) \leq C|t|^{-\alpha_{0}} \quad \text { for all } t>0 .
$$

We also allow $\alpha_{0}=1$ and in this case, we take $\gamma_{0}(t)=\delta(t)$ (the Dirac delta function).

Hypothesis 3.3. Regarding the spectral density $\mu(\xi)$, we assume that it satisfies one of the following two hypotheses.

(i) There are positive constants $\alpha_{i} \in(-1,0](i=1, \ldots, d)$ and $C$ such that

$$
\mu(\xi) \leq C \prod_{i=1}^{d}\left|\xi_{i}\right|^{\alpha_{i}} \quad \text { for all } \xi \in \mathbb{R}^{d} .
$$

We denote $\alpha=\alpha_{1}+\cdots+\alpha_{d}$ and we also assume $2 \alpha_{0}+\alpha<4$.

(ii) The space dimension $d=1$ and there are positive constants $\alpha>0, C$ such that

$$
\alpha+\alpha_{0}<3 / 2, \quad \text { and } \quad \mu(\xi) \leq C|\xi|^{\alpha} \quad \text { for all } \xi \in \mathbb{R} .
$$

Let us now state a new existence and uniqueness theorem for our equation of interest. 
Theorem 3.4. Suppose that the hypotheses 3.2 and 3.3 are satisfied and that the initial condition $u_{0}$ satisfies

$$
\int_{\mathbb{R}}\left[1+|\xi|^{\alpha / 2}\right] e^{-s|\xi|^{2}}\left|\hat{u}_{0}(\xi)\right| d \xi \leq C s^{-\beta}
$$

for some $\beta<1-\frac{\alpha_{0}}{2}$. Then there exists a unique solution to equation (1.1), that is, there is a unique process $u$ such that $p_{t-} .(x-\cdot) u$ is Skorohod integrable for any $(t, x) \in[0, T] \times \mathbb{R}^{d}$ and relation (3.1) holds true. Moreover, under the hypothesis 3.3 (ii) we have the following moment bounds for the solution:

$$
\mathbb{E}|u(t, x)|^{p} \leq C \exp \left\{c p^{\frac{3-\alpha}{1-\alpha}} t^{\frac{3-2 \alpha_{0}-\alpha}{1-\alpha}}\right\},
$$

where $C$ and $c$ are two constants independent of $p, t$ and $x$.

Remark 3.5. (i) It is known [9] that the theorem is true when the hypotheses 3.2 and 3.3 (i) hold. When $\gamma_{0}(t)$ is the Dirac delta function (namely, when the noise $\dot{W}$ is white in time) and when hypothesis 3.3 (i) holds, the theorem is also true ([8]).

(ii) Under hypotheses 3.2 and 3.3 (i), the moments bounds is given by (if the initial condition is bounded below by a positive constant)

$$
C \exp \left(C t^{\frac{4-2 \alpha_{0}-\alpha}{2-\alpha}} p^{\frac{4-\alpha}{2-\alpha}}\right) \leq \mathbb{E}\left[u_{t, x}^{p}\right] \leq C^{\prime} \exp \left(C^{\prime} t^{\frac{4-2 \alpha_{0}-\alpha}{2-\alpha}} p^{\frac{4-\alpha}{2-\alpha}}\right) .
$$

It appears that there are some discrepancy between (3.6) and (3.7). This kind of phenomena was also observed in [3].

Proof. We only need to show the theorem under the hypotheses 3.2 and 3.3 (ii) with $\alpha_{0} \in(0,1)$. If a solution $u$ is square integrable, then according to the general chaos expansion (2.6), $u(t, x)$ admits the following Wiener chaos expansion

$$
u(t, x)=\sum_{n=0}^{\infty} I_{n}\left(f_{n}(\cdot, t, x)\right),
$$

where for each $(t, x), f_{n}(\cdot, t, x)$ is a symmetric element in $\mathfrak{H}^{\otimes n}$. Hence, if one iterates (3.1), one can find an explicit formula for the kernels $f_{n}$ for $n \geq 1$.

$$
f_{n}\left(s_{1}, x_{1}, \ldots, s_{n}, x_{n}, t, x\right)=\frac{1}{n !} p_{t-s_{\sigma(n)}}\left(x-x_{\sigma(n)}\right) \cdots p_{s_{\sigma(2)}-s_{\sigma(1)}}\left(x_{\sigma(2)}-x_{\sigma(1)}\right) p_{s_{\sigma(1)}} u_{0}\left(x_{\sigma(1)}\right),
$$

where $\sigma$ denotes the permutation of $\{1,2, \ldots, n\}$ such that $0<s_{\sigma(1)}<\cdots<s_{\sigma(n)}<t$ (see also for instance, formula (4.4) in [6], formula (3.8) in [8], formula (3.3) in [9]). Then, to show the existence and uniqueness of the solution it suffices to prove that for all $(t, x)$ we have

$$
\sum_{n=0}^{\infty} n !\left\|f_{n}(\cdot, t, x)\right\|_{\mathfrak{H}^{\otimes n}}^{2}<\infty .
$$

The Fourier transform of $f_{n}(t, x)$ (with respect to the variables $x_{1}, \cdots, x_{n}$ in (3.9)) is

$$
\hat{f}_{n}\left(s_{1}, \xi_{1}, \ldots, s_{n}, \xi_{n}, t, x\right)=\frac{c^{n}}{n !} \int_{\mathbb{R}} g(t, x ; s, \xi, \zeta) \hat{u}_{0}(\zeta) e^{-\frac{s_{\sigma(1)}|\zeta|^{2}}{2}} d \zeta,
$$

where we denote $s_{\sigma(n+1)}=t$ by convention and

$$
g_{n}(t, x ; s, \xi, \zeta)=e^{-\frac{1}{2} s_{\sigma(1)}|\zeta|^{2}} \prod_{i=1}^{n} e^{-\frac{1}{2}\left(s_{\sigma(i+1)}-s_{\sigma(i)}\right)\left|\xi_{\sigma(i)}+\cdots+\xi_{\sigma(1)}-\zeta\right|^{2}} e^{-\iota x \cdot\left(\xi_{\sigma(n)}+\cdots+\xi_{\sigma(1)}-\zeta\right)} .
$$

Hence, we have

$$
\begin{aligned}
n !\left\|f_{n}(\cdot, t, x)\right\|_{\mathfrak{H}^{\otimes n}}^{2}=\frac{c^{2 n}}{n !} \int_{[0, t]^{2 n}} \int_{\mathbb{R}^{n}} \int_{\mathbb{R}^{2}} g(t, x ; s, \xi, \zeta) \bar{g}\left(t, x ; s^{\prime}, \xi, \zeta^{\prime}\right) \\
\quad \gamma_{0}\left(s-s^{\prime}\right) d s d s^{\prime} \mu\left(\xi_{1}\right) d \xi_{1} \cdots \mu\left(\xi_{n}\right) d \xi_{n} \hat{u}_{0}(\zeta) d \zeta \hat{u}_{0}\left(\zeta^{\prime}\right) d \zeta^{\prime},
\end{aligned}
$$

where $\gamma_{0}\left(s-s^{\prime}\right)=\prod_{i=1}^{n} \gamma_{0}\left(s_{i}-s_{i}^{\prime}\right)$. Using Cauchy-Schwarz inequality we get

$$
n !\left\|f_{n}(\cdot, t, x)\right\|_{\mathfrak{H}^{\otimes n}}^{2} \leq \frac{c^{2 n}}{n !} \int_{\mathbb{R}^{2}} \int_{[0, t]^{2 n}} \sqrt{\phi_{n}(s, \zeta) \phi_{n}\left(s^{\prime}, \zeta^{\prime}\right)} \gamma_{0}\left(s-s^{\prime}\right) d s d s^{\prime}\left|\hat{u}_{0}(\zeta)\right|\left|\hat{u}_{0}\left(\zeta^{\prime}\right)\right| d \zeta d \zeta^{\prime}
$$




$$
=\frac{c^{2 n}}{n !} \int_{\mathbb{R}^{2}} \int_{[0, t]^{2 n}} \psi_{n}(s) \psi_{n}\left(s^{\prime}\right) \gamma_{0}\left(s-s^{\prime}\right) d s d s^{\prime}
$$

where

$$
\phi_{n}(s, \zeta)=\int_{\mathbb{R}^{n}}\left|g_{n}(t, x ; s, \xi, \zeta)\right|^{2} \mu\left(\xi_{1}\right) d \xi_{1} \cdots \mu\left(\xi_{n}\right) d \xi_{n} \quad \text { and } \quad \psi_{n}(s)=\int_{\mathbb{R}} \sqrt{\phi_{n}(s, \zeta)}\left|\hat{u}_{0}(\zeta)\right| d \zeta .
$$

From (3.11) and the assumption 3.3, it follows that

$$
\phi_{n}(s, \zeta) \leq \int_{\mathbb{R}^{n}} e^{-s_{\sigma(1)}|\zeta|^{2}} \prod_{i=1}^{n} e^{-\left(s_{\sigma(i+1)}-s_{\sigma(i)}\right)\left|\xi_{\sigma(i)}+\cdots+\xi_{\sigma(1)}-\zeta\right|^{2}}\left|\xi_{1}\right|^{\alpha} \cdots\left|\xi_{n}\right|^{\alpha} d \xi_{1} \cdots d \xi_{n} .
$$

Making the change of variables $\eta_{i}:=\xi_{\sigma(i)}+\cdots+\xi_{\sigma(1)}-\zeta$, and using the trivial bound $\left|\eta_{i}-\eta_{i-1}\right|^{\alpha} \leq$ $\left|\eta_{i-1}\right|^{\alpha}+\left|\eta_{i}\right|^{\alpha}$, we obtain

$$
\begin{aligned}
\phi_{n}(s, \zeta) & \leq e^{-s_{\sigma(1)}|\zeta|^{2}} \int_{\mathbb{R}^{n}} \prod_{i=1}^{n} e^{-\left(s_{\sigma(i+1)}-s_{\sigma(i)}\right)\left|\eta_{i}\right|^{2}}\left|\eta_{1}+\zeta\right|^{\alpha} \prod_{i=2}^{n}\left|\eta_{i}-\eta_{i-1}\right|^{\alpha} d \eta_{1} \cdots d \eta_{n} \\
& \leq e^{-s_{\sigma(1)}|\zeta|^{2}} \int_{\mathbb{R}^{n}} \prod_{i=1}^{n} e^{-\left(s_{\sigma(i+1)}-s_{\sigma(i)}\right)\left|\eta_{i}\right|^{2}}\left(\left|\eta_{1}\right|^{\alpha}+|\zeta|^{\alpha}\right) \prod_{i=2}^{n}\left(\left|\eta_{i}\right|^{\alpha}+\left|\eta_{i-1}\right|^{\alpha}\right) d \eta_{1} \cdots d \eta_{n} .
\end{aligned}
$$

Expanding the product $\prod_{i=2}^{n}\left(\left|\eta_{i}\right|^{\alpha}+\left|\eta_{i-1}\right|^{\alpha}\right)$ in the above integral we obtain an expression of the form $\sum_{j \in D_{n}} \prod_{i=1}^{n}\left|\eta_{i}\right|^{j_{i}}$, where $D_{n}$ is a subset of multi-indices of $\left\{\left(j_{1}, \cdots, j_{n}\right): j_{i} \in\{0, \alpha, 2 \alpha\}\right\}$ of length $n-1$. The complete description of $D_{n}$ is not necessary. All we need are the following facts: $\operatorname{Card}\left(D_{n}\right)=2^{n-1}$ and for any $j \in D_{n}, j_{1} \in\{0, \alpha\}, j_{i} \in\{0, \alpha, 2 \alpha\}$ for $i=2, \ldots, n$ and

$$
|j| \equiv \sum_{i=1}^{n} j_{i}=(n-1) \alpha .
$$

This expansion yields the following bound for $\phi_{n}(s, \zeta)$.

$$
\begin{aligned}
\phi_{n}(s, \zeta) \leq|\zeta|^{\alpha} e^{-s_{\sigma(1)}|\zeta|^{2}} \sum_{\alpha \in D_{n}} \int_{\mathbb{R}^{n}} \prod_{i=1}^{n} e^{-\left(s_{\sigma(i+1)}-s_{\sigma(i)}\right)\left|\eta_{i}\right|^{2}} \prod_{i=1}^{n}\left|\eta_{i}\right|^{j_{i}} d \eta_{1} \cdots d \eta_{n} \\
\quad+e^{-s_{\sigma(1)}|\zeta|^{2}} \sum_{\alpha \in D_{n}} \int_{\mathbb{R}^{n}} \prod_{i=1}^{n} e^{-\left(s_{\sigma(i+1)}-s_{\sigma(i)}\right)\left|\eta_{i}\right|^{2}}\left|\eta_{1}\right|^{\alpha} \prod_{i=1}^{n}\left|\eta_{i}\right|^{j i} d \eta_{1} \cdots d \eta_{n} .
\end{aligned}
$$

Making the substitution $\xi_{i}=\left(s_{\sigma(i+1)}-s_{\sigma(i)}\right)^{1 / 2} \eta_{i}$ in the above integral, and noticing that $\int_{\mathbb{R}} e^{-\xi^{2}}|\xi|{ }^{j_{i}} d \xi$ is bounded by a constant for any $j_{i}>-1$, we see that

$$
\begin{aligned}
\phi_{n}(s, \zeta) \leq C|\zeta|^{\alpha} e^{-s_{\sigma(1)}|\zeta|^{2}} \sum_{\alpha \in D_{n}} \prod_{i=1}^{n}\left(s_{\sigma(i+1)}-s_{\sigma(i)}\right)^{-\frac{1}{2}\left(1+j_{i}\right)} \\
+C e^{-s_{\sigma(1)}|\zeta|^{2}} \sum_{\alpha \in D_{n}}\left(s_{\sigma(2)}-s_{\sigma(1)}\right)^{-\frac{\alpha+j_{1}}{2}} \prod_{i=2}^{n}\left(s_{\sigma(i+1)}-s_{\sigma(i)}\right)^{-\frac{1}{2}\left(1+j_{i}\right)} .
\end{aligned}
$$

Thus we have

$$
\begin{aligned}
\psi_{n}(s) \leq C \int_{\mathbb{R}}|\zeta|^{\alpha / 2} e^{-s_{\sigma(1)}|\zeta|^{2} / 2}\left|\hat{u}_{0}(\zeta)\right| d \zeta \sum_{\alpha \in D_{n}} \prod_{i=1}^{n}\left(s_{\sigma(i+1)}-s_{\sigma(i)}\right)^{-\frac{1}{4}\left(1+j_{i}\right)} \\
+C \int_{\mathbb{R}} e^{-s_{\sigma(1)}|\zeta|^{2} / 2}\left|\hat{u}_{0}(\zeta)\right| d \zeta \sum_{\alpha \in D_{n}}\left(s_{\sigma(2)}-s_{\sigma(1)}\right)^{-\frac{\alpha+j_{1}}{4}} \prod_{i=2}^{n}\left(s_{\sigma(i+1)}-s_{\sigma(i)}\right)^{-\frac{1}{4}\left(1+j_{i}\right)} .
\end{aligned}
$$

From the assumption on the initial condition (3.5) we see

$$
\psi_{n}(s) \leq C s_{\sigma(1)}^{-\beta} \sum_{\alpha \in D_{n}} \prod_{i=1}^{n}\left(s_{\sigma(i+1)}-s_{\sigma(i)}\right)^{-\frac{1}{4}\left(1+j_{i}\right)}
$$




$$
+C s_{\sigma(1)}^{-\beta} \sum_{\alpha \in D_{n}}\left(s_{\sigma(2)}-s_{\sigma(1)}\right)^{-\frac{\alpha+j_{1}}{4}} \prod_{i=2}^{n}\left(s_{\sigma(i+1)}-s_{\sigma(i)}\right)^{-\frac{1}{4}\left(1+j_{i}\right)} .
$$

Now we return to (3.12). From the Hardy-Littlewood inequality (see [6, Inequality (2.4)] or [12, Inequality $(1.5)])$, we see that

$$
n !\left\|f_{n}(\cdot, t, x)\right\|_{\mathfrak{H}^{\otimes n}}^{2} \leq \frac{c^{n}}{n !}\left(\int_{[0, t]^{n}} \psi_{n}(s)^{\frac{2}{2-\alpha_{0}}} d s\right)^{2-\alpha_{0}} .
$$

Plugging (3.14) into the above expression and uing the inequality $\left(a_{1}+\cdots+a_{m}\right)^{\alpha} \leq m^{\alpha}\left(a_{1}^{\alpha}+\cdots+a_{m}^{\alpha}\right)$ for all positive numbers $a_{1}, \cdots, a_{m}$ and positive $\alpha$, we end up with

$$
\begin{aligned}
n !\left\|f_{n}(\cdot, t, x)\right\|_{\mathfrak{H} \otimes n}^{2} \leq & \frac{c^{n}}{n !} \sum_{j \in D_{n}}\left(\int_{[0, t]^{n}} s_{\sigma(1)}^{-\frac{2 \beta}{2-\alpha_{0}}} \prod_{i=1}^{n}\left(s_{\sigma(i+1)}-s_{\sigma(i)}\right)^{-\frac{\left(1+j_{i}\right)}{4-2 \alpha_{0}}} d s\right)^{2-\alpha_{0}} \\
+ & \frac{c^{n}}{n !} \sum_{j \in D_{n}}\left(\int_{[0, t]^{n}} s_{\sigma(1)}^{-\frac{2 \beta}{2-\alpha_{0}}}\left(s_{\sigma(2)}-s_{\sigma(1)}\right)^{-\frac{\alpha+j_{1}}{4-2 \alpha_{0}}} \prod_{i=2}^{n}\left(s_{\sigma(i+1)}-s_{\sigma(i)}\right)^{-\frac{1+j_{i}}{4-2 \alpha_{0}}}\right)^{2-\alpha_{0}} \\
= & c^{n}(n !)^{1-\alpha_{0}} \sum_{j \in D_{n}}\left(\int_{T_{n}(t)} s_{\sigma(1)}^{-\frac{2 \beta}{2-\alpha_{0}}} \prod_{i=1}^{n}\left(s_{i+1}-s_{i}\right)^{-\frac{\left(1+j_{i}\right)}{4-2 \alpha_{0}}} d s\right)^{2-\alpha_{0}} \\
+c^{n}(n !)^{1-\alpha_{0}} & \sum_{j \in D_{n}}\left(\int_{T_{n}(t)} s_{1}^{-\frac{2 \beta}{2-\alpha_{0}}}\left(s_{2}-s_{1}\right)^{-\frac{\alpha+j_{1}}{4-2 \alpha_{0}}} \prod_{i=2}^{n}\left(s_{i+1}-s_{i}\right)^{-\frac{1+j_{i}}{4-2 \alpha_{0}}} \cdot\right)^{2-\alpha_{0}} .
\end{aligned}
$$

From our condition on $\beta$, we see that $\frac{2 \beta}{2-\alpha_{0}}<1$. For $i \geq 2$, the maximum value of $j_{i}$ is $2 \alpha$ and in this case

$$
\frac{1+j_{i}}{4-2 \alpha_{0}}=\frac{1+2 \alpha}{4-2 \alpha_{0}}<1
$$

Since $j_{1} \leq \alpha$,

$$
\frac{\alpha+j_{1}}{4-2 \alpha_{0}} \leq \frac{2 \alpha}{4-2 \alpha_{0}}<1
$$

It follows that all the integrals appeared in the above multiple integrals are finite. Using Lemma 2.1, we see that

$$
\begin{aligned}
\int_{T_{n}(t)} s_{\sigma(1)}^{-\frac{2 \beta}{2-\alpha_{0}}} \prod_{i=1}^{n}\left(s_{i+1}-s_{i}\right)^{-\frac{\left(1+j_{i}\right)}{4-2 \alpha_{0}}} d s & \leq \frac{c^{n} t^{\sum_{i=1}^{n}\left(1-\frac{1+j_{i}}{4-2 \alpha_{0}}\right)+1-\frac{2 \beta}{2-\alpha_{0}}}}{\Gamma\left(\sum_{i=1}^{n}\left(1-\frac{1+j_{i}}{4-2 \alpha_{0}}\right)+1-\frac{2 \beta}{2-\alpha_{0}}+1\right)} \\
& \leq c^{n} t^{\tilde{\alpha}_{0}} t^{\frac{\left(3-2 \alpha_{0}-\alpha\right) n}{4-2 \alpha_{0}}}(n !)^{-\frac{3-2 \alpha_{0}-\alpha}{4-2 \alpha_{0}}} .
\end{aligned}
$$

In the same way we have

$$
\int_{T_{n}(t)} s_{1}^{-\frac{2 \beta}{2-\alpha_{0}}}\left(s_{2}-s_{1}\right)^{-\frac{\alpha+j_{1}}{4-2 \alpha_{0}}} \prod_{i=2}^{n}\left(s_{i+1}-s_{i}\right)^{-\frac{1+j_{i}}{4-2 \alpha_{0}}} d s \leq c^{n} t^{\tilde{\alpha}_{1}} t^{\frac{\left(3-2 \alpha_{0}-\alpha\right) n}{4-2 \alpha_{0}}}(n !)^{-\frac{3-2 \alpha_{0}-\alpha}{4-2 \alpha_{0}}} .
$$

Here $\tilde{\alpha}_{0}$ and $\tilde{\alpha}_{1}$ are two constants, independent of $n$. Inserting the above two bounds into (3.15), we see that

$$
n !\left\|f_{n}(\cdot, t, x)\right\|_{\mathfrak{H} \otimes n}^{2} \leq c^{n} t^{\tilde{\alpha}_{2}}(n !)^{1-\alpha_{0}-\frac{3-2 \alpha_{0}-\alpha}{2}} t^{\frac{3-2 \alpha_{0}-\alpha}{2} n}=c^{n} t^{\tilde{\alpha}_{2}}(n !)^{-\frac{1-\alpha}{2}} t^{\frac{3-2 \alpha_{0}-\alpha}{2} n} .
$$

From hypercontractivity ([4] and [13], we see that

$$
\left\|I_{n}\left(f_{n}(t, x)\right)\right\|_{p} \leq \sqrt{p-1}^{n}\left\|I_{n}\left(f_{n}(t, x)\right)\right\|_{2} \leq c^{n} \sqrt{p}^{n} t^{\tilde{\alpha}_{2} / 2}(n !)^{-\frac{1-\alpha}{4}} t^{\frac{3-2 \alpha_{0}-\alpha}{4} n} .
$$

Applying the estimate (2.7), we obtain

$$
\|u(t, x)\|_{p} \leq \sum_{n=0}^{\infty} p^{\frac{n}{2}} c^{n} t^{\tilde{\alpha}_{2} / 2}(n !)^{-\frac{1-\alpha}{4}} t^{\frac{3-2 \alpha_{0}-\alpha}{4} n} \leq C \exp \left\{c p^{\frac{2}{1-\alpha}} t^{\frac{3-2 \alpha_{0}-\alpha}{1-\alpha}}\right\},
$$

which implies (3.6). 


\section{HÖlder AND JOINT HÖLDER CONTINUity}

In this section, we shall study the joint Hölder continuity of the solution $u(t, x)$ to $(1.1)$. The following lemma will be useful later.

Lemma 4.1. Let $\mu$ satisfy the hypothesis 3.3 (i). For any $\beta \geq 0$ and $s>0$, we have

$$
\sup _{\zeta \in \mathbb{R}^{d}} \int_{\mathbb{R}^{d}} e^{-2 s|\xi-\zeta|^{2}}|\xi-\zeta|^{\beta} \mu(\xi) d \xi \lesssim s^{-\frac{\alpha+\beta}{2}} .
$$

Proof. Put $C_{\beta}:=\sup _{x>0} e^{-x} x^{\beta}$, which is a finite constant. We note that

$$
\begin{aligned}
\int_{\mathbb{R}^{d}} e^{-2 s|\xi-\zeta|^{2}}|\xi-\zeta|^{\beta} \mu(\xi) d \xi & \leq C_{\beta} s^{-\frac{\beta}{2}} \int_{\mathbb{R}^{d}} e^{-s|\xi-\zeta|^{2}} \mu(\xi) d \xi \\
& =C_{\beta} s^{-\frac{\beta}{2}} \int_{\mathbb{R}^{d}} e^{-s|\xi-\zeta|^{2}}\left|\xi_{1}\right|^{\alpha_{1}} d \xi_{1} \cdots\left|\xi_{d}\right|^{\alpha_{d}} d \xi_{d} .
\end{aligned}
$$

From Lemma A.1 of [11], we have

$$
\int_{\mathbb{R}^{d}} e^{-s|\xi-\zeta|^{2}}\left|\xi_{1}\right|^{\alpha_{1}} d \xi_{1} \cdots\left|\xi_{d}\right|^{\alpha_{d}} d \xi_{d} \lesssim s^{-\frac{\alpha}{2}}
$$

This implies the result.

To simplify notation we denote $u_{n}(t, x)=I_{n, t}\left(f_{n}(t, x, \cdot)\right)$, where $f_{n}$ is the $n$-th chaos kernel defined in (3.9) and we explicitly express the dependence of the multiple integral of $f_{n}$ on $t$ :

$$
I_{n, t}\left(f_{n}(t, x)\right)=\int_{[0, t]^{n}} f_{n}\left(t, x ; s_{s}, \cdots, s_{n}, x_{1}, \cdots, x_{n}\right) W\left(d s_{s}, d x_{1}\right) \cdots W\left(d s_{n}, d x_{n}\right) .
$$

Thus, the solution $u(t, x)$ can be written as its chaos expansion: $u(t, x)=\sum_{n=0}^{\infty} u_{n}(t, x)$.

Proposition 4.2. Assuming that hypothesis 3.2 holds and $u_{0}$ satisfies (3.5).

(i) Let the hypothesis 3.3 (i) be satisfied and let $\bar{\alpha}_{0}$ and $\bar{\alpha}$ be in $[0,1]$ such that

$$
2 \bar{\alpha}_{0}+\bar{\alpha}<2-\alpha_{0}-\frac{\alpha}{2} .
$$

[It is easy to see the right hand side is positive under our assumption.] Then, there is a positive constant $c$ such that for every $t \geq r \geq 0$ and $x, y \in \mathbb{R}^{d}$

$$
\mathbb{E}\left|u_{n}(t, y)-u_{n}(r, y)-u_{n}(t, x)+u_{n}(r, x)\right|^{2} \leq c^{n}(n !)^{\frac{\alpha}{2}-1}|t-r|^{2 \bar{\alpha}_{0}}|x-y|^{2 \bar{\alpha}} t^{\frac{4-2 \alpha_{0}-\alpha}{2} n} .
$$

(ii) Let the hypothesis 3.3 (ii) be satisfied and let $\bar{\alpha}_{0}$ and $\bar{\alpha}$ be in $[0,1]$ such that

$$
2 \bar{\alpha}_{0}+\bar{\alpha}<\frac{3-2 \alpha_{0}-\alpha}{2} .
$$

Then, there is a positive constant $c$ such that for every $t \geq r \geq 0$ and $x, y \in \mathbb{R}^{d}$

$$
\mathbb{E}\left|u_{n}(t, y)-u_{n}(r, y)-u_{n}(t, x)+u_{n}(r, x)\right|^{2} \leq c^{n}(n !)^{\frac{\alpha-1}{2}}|t-r|^{2 \bar{\alpha}_{0}}|x-y|^{2 \bar{\alpha}} t^{\frac{3-2 \alpha_{0}-\alpha}{2} n} .
$$

Proof. We follow the notation in the proof of Theorem 3.4 and divide the proof into several steps.

Step 1. For every $s=\left(s_{1}, \cdots, s_{n}\right) \in[0, t]^{n}$, the Fourier transform of $f_{n}\left(t, x, s_{1}, x_{1}, \ldots, s_{n}, x_{n}\right)$ with respect to the spatial variables $\left(x_{1}, \ldots, x_{n}\right)$, denoted by $\hat{f}_{n}(t, x, s, \xi)$ is

$$
\begin{aligned}
\hat{f}_{n}(t, x) & =\hat{f}_{n}\left(t, x, s_{1}, \xi_{1}, \ldots, s_{n}, \xi_{n}\right) \\
& =\frac{c^{n}}{n !} \int_{\mathbb{R}^{d}} \prod_{i=1}^{n} e^{-\frac{1}{2}\left(s_{\sigma(i+1)}-s_{\sigma(i)}\right)\left|\xi_{\sigma(i)}+\cdots+\xi_{\sigma(1)}-\zeta\right|^{2}} e^{-\imath x \cdot\left(\xi_{\sigma(n)}+\cdots+\xi_{\sigma(1)}-\zeta\right)} \hat{u}_{0}(\zeta) e^{-\frac{s_{\sigma(1)}|\zeta|^{2}}{2}} d \zeta,
\end{aligned}
$$

where $\sigma$ is a permutation of $\{1, \ldots, n\}$ such that $s_{\sigma(1)} \leq \cdots \leq s_{\sigma(n)}$ and we have set $s_{\sigma(n+1)}=t$. We use the following notation

$$
\eta_{i}=\xi_{\sigma(i)}+\cdots+\xi_{\sigma(1)} \quad \text { and } \quad g_{n}(s, \eta, \zeta)=\prod_{i=1}^{n-1} e^{-\frac{1}{2}\left(s_{\sigma(i+1)}-s_{\sigma(i)}\right)\left|\eta_{i}-\zeta\right|^{2}}
$$


Thus, we can rewrite

$$
\hat{f}_{n}(t, x)=\frac{c^{n}}{n !} \int_{\mathbb{R}^{d}} e^{-\frac{1}{2}\left(t-s_{\sigma(n)}\right)\left|\eta_{n}-\zeta\right|^{2}-\imath x \cdot\left(\eta_{n}-\zeta\right)} g_{n}(s, \eta, \zeta) \hat{u}_{0}(\zeta) e^{-\frac{s_{\sigma(1)}|\zeta|^{2}}{2}} d \zeta .
$$

We denote $\Delta_{r, t}^{n}=[0, t]^{n} \backslash[0, r]^{n}$ and apply triangle inequality to get

$$
\mathbb{E}\left|u_{n}(t, x)-u_{n}(t, y)-u_{n}(r, x)+u_{n}(r, y)\right|^{2} \leq 2 A_{n}+2 B_{n},
$$

where

$$
A_{n}=\mathbb{E}\left|I_{n, r}\left(f_{n}(t, x, \cdot)-f_{n}(t, y, \cdot)-f_{n}(r, x, \cdot)+f_{n}(r, y, \cdot)\right)\right|^{2}
$$

and

$$
B_{n}=\mathbb{E}\left|I_{n, t}\left(\left[f_{n}(t, x, \cdot)-f_{n}(t, y, \cdot)\right] \mathbf{1}_{\Delta_{r, t}^{n}}\right)\right|^{2} .
$$

Step 2. For every $s, s^{\prime} \in[0, r]^{n}$, we denote

$$
\begin{aligned}
\phi\left(s, s^{\prime}\right)=\frac{1}{(2 \pi)^{n d}} & \int_{\mathbb{R}^{n d}}\left(\hat{f}_{n}(t, x, s, \xi)-\hat{f}_{n}(t, y, s, \xi)-\hat{f}_{n}(r, x, s, \xi)+\hat{f}_{n}(r, y, s, \xi)\right) \\
& \times \overline{\left(\hat{f}_{n}\left(t, x, s^{\prime}, \xi\right)-\hat{f}_{n}\left(t, y, s^{\prime}, \xi\right)-\hat{f}_{n}\left(r, x, s^{\prime}, \xi\right)+\hat{f}_{n}\left(r, y, s^{\prime}, \xi\right)\right)} \mu\left(\xi_{1}\right) d \xi_{1} \cdots \mu\left(\xi_{n}\right) d \xi_{n}
\end{aligned}
$$

and

$$
\phi_{1}(s)=\frac{1}{(2 \pi)^{n d}} \int_{\mathbb{R}^{n d}}\left|\hat{f}_{n}(t, x, s, \xi)-\hat{f}_{n}(t, y, s, \xi)-\hat{f}_{n}(r, x, s, \xi)+\hat{f}_{n}(r, y, s, \xi)\right|^{2} \mu\left(\xi_{1}\right) d \xi_{1} \cdots \mu\left(\xi_{n}\right) d \xi_{n} .
$$

Applying Itô isometry of Wiener chaos and Cauchy-Schwarz, we have

$$
A_{n}=n ! \int_{[0, r]^{n}} \int_{[0, r]^{n}} \phi\left(s, s^{\prime}\right) \prod_{j=1}^{n} \gamma_{0}\left(s_{j}-s_{j}^{\prime}\right) d s d s^{\prime} \leq n ! \int_{[0, r]^{n}} \int_{[0, r]^{n}}\left[\phi_{1}(s) \phi_{1}\left(s^{\prime}\right)\right]^{\frac{1}{2}} \prod_{j=1}^{n} \gamma_{0}\left(s_{j}-s_{j}^{\prime}\right) d s d s^{\prime} .
$$

Using the Hardy-Littlewood inequality (see [6, Inequality (2.4)] or [12, Inequality (1.5)]) we see that

$$
A_{n} \lesssim c^{n} n !\left(\int_{[0, r]^{n}}\left|\phi_{1}(s)\right|^{\frac{1}{2-\alpha_{0}}} d s\right)^{2-\alpha_{0}}
$$

Step 3. We estimate bound $A_{n}$ under hypothesis 3.3 (i). Observe that for every $s \in[0, r]^{n}$,

$$
\begin{aligned}
& \hat{f}_{n}(t, x, s, \xi)-\hat{f}_{n}(t, y, s, \xi)-\hat{f}_{n}(r, x, s, \xi)+\hat{f}_{n}(r, y, s, \xi) \\
& =\frac{c^{n}}{n !} \int_{\mathbb{R}^{d}} e^{-\frac{1}{2}\left(r-s_{\sigma(n)}\right)\left|\eta_{n}-\zeta\right|^{2}-\imath y \cdot\left(\eta_{n}-\zeta\right)}\left[e^{-\frac{1}{2}(t-r)\left|\eta_{n}-\zeta\right|^{2}}-1\right]\left[e^{-\imath(x-y) \cdot\left(\eta_{n}-\zeta\right)}-1\right] g_{n}(s, \eta, \zeta) \hat{u}_{0}(\zeta) e^{-\frac{s_{\sigma(1)}||^{2}}{2}} d \zeta .
\end{aligned}
$$

Applying Jensen's inequality with respect to the measure $\left|\hat{u}_{0}(\zeta)\right| e^{-\frac{s_{\sigma(1)}|\zeta|^{2}}{2}} d \zeta$, we obtain

$$
\begin{aligned}
& \left|\hat{f}_{n}(t, x, s, \xi)-\hat{f}_{n}(t, y, s, \xi)-\hat{f}_{n}(r, x, s, \xi)+\hat{f}_{n}(r, y, s, \xi)\right|^{2} \\
& \leq \frac{c^{n}}{(n !)^{2}} \int_{\mathbb{R}^{d}}\left|\hat{u}_{0}(\zeta)\right| e^{-\frac{s_{\sigma(1)}|\zeta|^{2}}{2}} d \zeta \times \int_{\mathbb{R}^{d}} e^{-\left(r-s_{\sigma(n)}\right)\left|\eta_{n}-\zeta\right|^{2}} \\
& \quad\left|e^{-\frac{1}{2}(t-r)\left|\eta_{n}-\zeta\right|^{2}}-1\right|^{2}\left|e^{-\imath(y-x) \cdot\left(\eta_{n}-\zeta\right)}-1\right|^{2}\left|g_{n}(s, \eta, \zeta)\right|^{2}\left|\hat{u}_{0}(\zeta)\right| e^{-\frac{s_{\sigma(1)}|\zeta|^{2}}{2}} d \zeta .
\end{aligned}
$$

Applying the elementary estimates

$$
\left|e^{-\frac{1}{2}(t-r)\left|\eta_{n}-\zeta\right|^{2}}-1\right| \lesssim|t-r|^{\bar{\alpha}_{0}}\left|\eta_{n}-\zeta\right|^{2 \bar{\alpha}_{0}}, \quad\left|e^{-\imath(y-x) \cdot\left(\eta_{n}-\zeta\right)}-1\right| \lesssim|y-x|^{\bar{\alpha}}\left|\eta_{n}-\zeta\right|^{\bar{\alpha}}
$$

for any $\tilde{\alpha}_{0}, \tilde{\alpha} \in[0,1]$ and noticing $(3.5)$, we see that

$$
\begin{aligned}
\phi_{1}(s) \lesssim & \frac{c^{n}}{(n !)^{2}}|t-r|^{2 \bar{\alpha}_{0}}|x-y|^{2 \bar{\alpha}} s_{\sigma(1)}^{-\beta} \int_{\mathbb{R}^{d}} \int_{\mathbb{R}^{n d}} e^{-\left(r-s_{\sigma(n)}\right)\left|\eta_{n}-\zeta\right|^{2}} \\
& \left|\eta_{n}-\zeta\right|^{4 \bar{\alpha}_{0}+2 \bar{\alpha}}\left|g_{n}(s, \eta, \zeta)\right|^{2} \mu\left(\xi_{1}\right) d \xi_{1} \cdots \mu\left(\xi_{n}\right) d \xi_{n}\left|\hat{u}_{0}(\zeta)\right| e^{-\frac{s_{\sigma(1)}|\zeta|^{2}}{2}} d \zeta .
\end{aligned}
$$


To estimate the integral above, we first consider the integration with respect to $\xi_{\sigma(n)}$. Noting that $g_{n}$ does not depend on $\xi_{\sigma(n)}$, we can apply Lemma 4.1 to get

$$
\int_{\mathbb{R}^{d}} e^{-\left(r-s_{\sigma(n)}\right)\left|\eta_{n}-\zeta\right|^{2}}\left|\eta_{n}-\zeta\right|^{4 \bar{\alpha}_{0}+2 \bar{\alpha}} \mu\left(\xi_{\sigma(n)}\right) d \xi_{\sigma(n)} \lesssim\left(r-s_{\sigma(n)}\right)^{-2 \bar{\alpha}_{0}-\bar{\alpha}-\frac{\alpha}{2}},
$$

where the implied constant is independent of $\zeta$ and $\xi_{1}, \ldots, \xi_{\sigma(n-1)}$. Next, we integrate the variable $\xi_{\sigma(n-1)}$. Due to the factor $e^{-\left(s_{\sigma(n)-s_{\sigma(n-1)}}\right)\left|\eta_{n-1}-\zeta\right|^{2}}$ in $g_{n}$, this amounts to estimate an analogous integral but with $\bar{\alpha}_{0}=\bar{\alpha}=0$. We continue this way until all the variables $\xi_{\sigma(1)}, \ldots, \xi_{\sigma(n)}$ are integrated out. The last integral is $\int_{\mathbb{R}^{d}} e^{-\frac{1}{2} s_{\sigma(1)}|\zeta|^{2}}\left|\hat{u}_{0}(\zeta)\right| d \zeta$ which is controlled by (3.5). Therefore, we have

$$
\phi_{1}(s) \lesssim \frac{c^{n}}{(n !)^{2}}|t-r|^{2 \bar{\alpha}_{0}}|x-y|^{2 \bar{\alpha}}\left(r-s_{\sigma(n)}\right)^{-2 \bar{\alpha}_{0}-\bar{\alpha}-\frac{\alpha}{2}} \prod_{j=1}^{n-1}\left(s_{\sigma(j+1)}-s_{\sigma(j)}\right)^{-\frac{\alpha}{2}} s_{\sigma(1)}^{-2 \beta} .
$$

Together with (4.7), we obtain

$$
\begin{aligned}
A_{n} & \lesssim \frac{c^{n}}{n !}|t-r|^{2 \bar{\alpha}_{0}}|x-y|^{2 \bar{\alpha}}\left[\int_{[0, r]^{n}}\left(r-s_{\sigma(n)}\right)^{-\frac{2 \bar{\alpha}_{0}+\bar{\alpha}+\frac{\alpha}{2}}{2-\alpha_{0}}} \prod_{j=1}^{n-1}\left(s_{\sigma(j+1)}-\sigma_{\sigma(j)}\right)^{-\frac{\alpha}{2\left(2-\alpha_{0}\right)}} s_{\sigma(1)}^{-\frac{2 \beta}{2-\alpha_{0}}} d s\right]^{2-\alpha_{0}} \\
& \lesssim c^{n}(n !)^{1-\alpha_{0}}|t-r|^{2 \bar{\alpha}_{0}}|x-y|^{2 \bar{\alpha}}\left[\int_{T_{n}(r)}\left(r-s_{n}\right)^{-\frac{2 \bar{\alpha}_{0}+\bar{\alpha}+\frac{\alpha}{2}}{2-\alpha_{0}}} \prod_{j=1}^{n-1}\left(s_{j+1}-s_{j}\right)^{-\frac{\alpha}{2\left(2-\alpha_{0}\right)}} s_{1}^{-\frac{2 \beta}{2-\alpha_{0}}} d s\right]^{2-\alpha_{0}}
\end{aligned}
$$

where $T_{n}(r)=\left\{\left(s_{1}, \ldots, s_{n}\right): s_{1}<\cdots<s_{n}<r\right\}$. Using the condition (4.4) and Lemma 2.1, we see that the integration over $T_{n}(r)$ above is finite and is bounded by

$$
r^{n \frac{2-\alpha_{0}-\frac{\alpha}{2}}{2-\alpha_{0}}-\frac{2 \bar{\alpha}_{0}+\bar{\alpha}+2 \beta}{2-\alpha_{0}}}\left[\Gamma\left(n \frac{2-\alpha_{0}-\frac{\alpha}{2}}{2-\alpha_{0}}+1\right)\right]^{-1} .
$$

Using Stirling formula, we have

$$
\begin{aligned}
A_{n} & \lesssim c^{n}|t-r|^{2 \bar{\alpha}_{0}}|x-y|^{2 \bar{\alpha}} r^{n\left(2-\alpha_{0}-\frac{\alpha}{2}\right)-2 \bar{\alpha}_{0}-\bar{\alpha}-2 \beta} \frac{(n !)^{1-\alpha_{0}}}{\Gamma\left(n \frac{2-\alpha_{0}-\frac{\alpha}{2}}{2-\alpha_{0}}+1\right)^{2-\alpha_{0}}} \\
& \lesssim c^{n}|t-r|^{2 \bar{\alpha}_{0}}|x-y|^{2 \bar{\alpha}} r^{n\left(2-\alpha_{0}-\frac{\alpha}{2}\right)-2 \bar{\alpha}_{0}-\bar{\alpha}-2 \beta} \frac{(n !)^{1-\alpha_{0}}}{\Gamma\left(n\left(2-\alpha_{0}-\frac{\alpha}{2}\right)+1\right)} \\
& \lesssim c^{n}|t-r|^{2 \bar{\alpha}_{0}}|x-y|^{2 \bar{\alpha}} r^{n\left(2-\alpha_{0}-\frac{\alpha}{2}\right)-2 \bar{\alpha}_{0}-\bar{\alpha}-2 \beta}(n !)^{\frac{\alpha}{2}-1} .
\end{aligned}
$$

Step 4. Now we consider $A_{n}$ for $d=1$ and $\alpha>0$. In this case the inequality (4.8) becomes

$$
\begin{aligned}
\phi_{1}(s) \lesssim & \frac{c^{n}}{(n !)^{2}}|t-r|^{2 \bar{\alpha}_{0}}|x-y|^{2 \bar{\alpha}} s_{\sigma(1)}^{-\beta} \int_{\mathbb{R}} \int_{\mathbb{R}^{n}} e^{-\left(r-s_{\sigma(n)}\right)\left|\eta_{n}-\zeta\right|^{2}}\left|\eta_{n}-\zeta\right|^{4 \bar{\alpha}_{0}+2 \bar{\alpha}} \\
& \prod_{i=1}^{n-1} e^{-\left(s_{\sigma(i+1)}-s_{\sigma(i)}\right)\left|\eta_{i}-\zeta\right|^{2}} \prod_{i=1}^{n}\left|\xi_{i}\right|^{\alpha} d \xi_{1} \cdots d \xi_{n}\left|\hat{u}_{0}(\zeta)\right| e^{-\frac{s_{\sigma(1)}|\zeta|^{2}}{2}} d \zeta .
\end{aligned}
$$

Making substitution $\eta_{i}-\zeta=\xi_{\sigma(i)}+\cdots+\xi_{s(\sigma(1)}-\zeta=z_{i}$, we have

$$
\begin{aligned}
\phi_{1}(s) \lesssim & \frac{c^{n}}{(n !)^{2}}|t-r|^{2 \bar{\alpha}_{0}}|x-y|^{2 \bar{\alpha}} s_{\sigma(1)}^{-\beta} \int_{\mathbb{R}} \int_{\mathbb{R}^{n}} e^{-\left(r-s_{\sigma(n)}\right)\left|z_{n}\right|^{2}} \prod_{i=1}^{n-1} e^{-\left(s_{\sigma(i+1)}-s_{\sigma(i)}\right)\left|z_{i}\right|^{2}} \\
& \left|z_{n}\right|^{4 \bar{\alpha}_{0}+2 \bar{\alpha}}\left|z_{1}+\zeta\right|^{\alpha} \prod_{i=2}^{n}\left|z_{i}-z_{i-1}\right|^{\alpha} d z_{1} \cdots d z_{n}\left|\hat{u}_{0}(\zeta)\right| e^{-\frac{s_{\sigma(1)}|\zeta|^{2}}{2}} d \zeta \\
\lesssim & \frac{c^{n}}{(n !)^{2}}|t-r|^{2 \bar{\alpha}_{0}}|x-y|^{2 \bar{\alpha}} s_{\sigma(1)}^{-\beta} \int_{\mathbb{R}} \int_{\mathbb{R}^{n}} e^{-\left(r-s_{\sigma(n)}\right)\left|z_{n}\right|^{2}} \prod_{i=1}^{n-1} e^{-\left(s_{\sigma(i+1)}-s_{\sigma(i)}\right)\left|z_{i}\right|^{2}} \\
& \left|z_{n}\right|^{4 \bar{\alpha}_{0}+2 \bar{\alpha}}\left(\left|z_{1}\right|^{\alpha}+|\zeta|^{\alpha}\right) \prod_{i=2}^{n}\left(\left|z_{i}\right|^{\alpha}+\left|z_{i-1}\right|^{\alpha}\right) d z_{1} \cdots d z_{n}\left|\hat{u}_{0}(\zeta)\right| e^{-\frac{s_{\sigma(1)}|\zeta|^{2}}{2}} d \zeta .
\end{aligned}
$$


As for (3.13), we expand $\left(\left|z_{1}\right|^{\alpha}+|\zeta|^{\alpha}\right) \prod_{i=2}^{n}\left(\left|z_{i}\right|^{\alpha}+\left|z_{i-1}\right|^{\alpha}\right)$ and integrate $d z_{1} \cdots d z_{n} d \zeta$ to obtain

$$
\phi_{1}(s) \leq \frac{c^{n}}{(n !)^{2}}|t-r|^{2 \bar{\alpha}_{0}}|x-y|^{2 \bar{\alpha}} s_{\sigma(1)}^{-2 \beta}\left(r-s_{\sigma(n)}\right)^{-2 \tilde{\alpha}_{0}-\tilde{\alpha}} \sum_{j \in D_{n}} \prod_{i=1}^{n}\left(s_{\sigma(i+1)}-s_{\sigma(i)}\right)^{-\frac{1}{2}\left(1+j_{i}\right)} .
$$

The set $D_{n}$ is similar to the one defined in Section 3 with a minor difference. More specifically, $D_{n}$ is a set of multi-indices with the following properties: $\operatorname{Card}\left(D_{n}\right)=2^{n-1}$, for each $j=\left(j_{1}, \ldots, j_{n}\right) \in D_{n}, j_{n} \in\{0, \alpha\}$, $j_{i} \in\{0, \alpha, 2 \alpha\}$ and

$$
|j| \equiv \sum_{i=1}^{n} j_{i}=(n-1) \alpha .
$$

Similar to the estimates (3.15)-(3.16) if we assume $2 \tilde{\alpha}_{0}+\tilde{\alpha}<\frac{3-2 \alpha_{0}-\alpha}{2}$, then we have

$$
\begin{aligned}
A_{n} \leq & c^{n}(n !)^{1-\alpha_{0}}|t-r|^{2 \tilde{\alpha}_{0}}|x-y|^{2 \tilde{\alpha}} \\
& \sum_{j \in D_{n}}\left[\int_{[0, r]^{n d}}\left(s_{\sigma(1)}^{-2 \beta}\left(r-s_{\sigma(n)}\right)^{-2 \tilde{\alpha}_{0}-\tilde{\alpha}-\frac{1+\alpha}{2}} \sum_{j \in D_{n}} \prod_{i=1}^{n-1}\left(s_{\sigma(i+1)}-s_{\sigma(i)}\right)^{-\frac{1}{2}\left(1+j_{i}\right)}\right)^{\frac{1}{2-\alpha_{0}}}\right]^{2-\alpha_{0}} \\
& \leq c^{n}(n !)^{\frac{\alpha-1}{2}}|t-r|^{2 \tilde{\alpha}_{0}}|x-y|^{2 \tilde{\alpha}} t^{\tilde{\alpha}_{3}} t^{\frac{3-2 \alpha_{0}-\alpha}{2} n} .
\end{aligned}
$$

Step 5. We estimate $B_{n}$ under hypothesis 3.3 (i). As in deriving (4.7), we apply Itô isometry of Wiener chaos, Cauchy-Schwarz inequality and Hardy-Littlewood inequality to obtain a similar inequality to (4.7). Namely,

where

$$
B_{n} \leq c^{n} n !\left(\int_{\Delta_{r, t}^{n}}\left|\psi_{1}(s)\right|^{\frac{1}{2-\alpha_{0}}} d s\right)^{2-\alpha_{0}}
$$

$$
\psi_{1}(s)=\frac{1}{(2 \pi)^{n d}} \int_{\mathbb{R}^{n d}}\left|\hat{f}_{n}(t, x, s, \xi)-\hat{f}_{n}(t, y, s, \xi)\right|^{2} \mu\left(\xi_{1}\right) d \xi_{1} \cdots \mu\left(\xi_{n}\right) d \xi_{n} .
$$

Similar to (4.13), we have

$$
\begin{aligned}
& \psi_{1}(s) \\
& \leq \frac{c^{n}}{(n !)^{2}}|x-y|^{2 \tilde{\alpha}} s_{\sigma(1)}^{-\beta} \int_{\mathbb{R}(n+1) d}\left|\eta_{n}-\zeta\right|^{2 \tilde{\alpha}} e^{-\left(t-s_{\sigma(n)}\right)\left|\eta_{n}-\zeta\right|^{2}}\left|g_{n}(s, \eta, \zeta)\right| \mu\left(\xi_{1}\right) d \xi_{1} \cdots \mu\left(\xi_{n}\right) d \xi_{n}\left|\hat{u}_{0}(\zeta)\right| e^{-\frac{s_{\sigma(1)}|\zeta|^{2}}{2}} d \zeta \\
& \leq \frac{c^{n}}{(n !)^{2}}|x-y|^{2 \tilde{\alpha}} s_{\sigma(1)}^{-2 \beta}\left(t-s_{\sigma(n)}\right)^{-\frac{\alpha}{2}-\tilde{\alpha}} \prod_{i=1}^{n-1}\left(s_{\sigma(i+1)}-s_{\sigma(i+1)}\right)^{-\frac{\alpha}{2}}
\end{aligned}
$$

Thus we have

$$
B_{n} \leq \frac{c^{n}}{n !}|x-y|^{2 \tilde{\alpha}}\left(\int_{\Delta_{r, t}^{n}}\left(s_{\sigma(1)}^{-2 \beta}\left(t-s_{\sigma(n)}\right)^{-\frac{\alpha}{2}-\tilde{\alpha}} \prod_{i=1}^{n-1}\left(s_{\sigma(i+1)}-s_{\sigma(i+1)}\right)^{-\frac{\alpha}{2}}\right)^{\frac{1}{2-\alpha_{0}}} d s\right)^{2-\alpha_{0}},
$$

The integrating region $\Delta_{r, t}^{n}$ can be decomposed as $\Delta_{r, t}^{n}=\cup_{\sigma} \cup_{0 \leq k \leq n} T_{n, \sigma, k, r, t}$, where $\sigma$ is a permutation of $\{1,2, \cdots, n\}$ and

$$
T_{n, \sigma, k, r, t}=\left\{0<s_{\sigma(1)}<\cdots<s_{\sigma(k)}<r<s_{\sigma(k+1)}<\cdots<s_{\sigma(n)}<t\right\} .
$$

When $\sigma$ is the identity, we denote $T_{n, k, r, t}=T_{n, \sigma, k, r, t}$. Thus by symmetry

$$
B_{n} \leq(n !)^{1-\alpha_{0}} c^{n}|x-y|^{2 \tilde{\alpha}}\left(\sum_{k=0}^{n} \int_{T_{n, k, r, t}}\left(s_{1}^{-2 \beta}\left(t-s_{n}\right)^{-\frac{\alpha}{2}-\tilde{\alpha}} \prod_{i=1}^{n-1}\left(s_{i+1}-s_{i}\right)^{-\frac{\alpha}{2}}\right)^{\frac{1}{2-\alpha_{0}}} d s\right)^{2-\alpha_{0}},
$$

For each $k$, the multiple integral over $T_{n, k, r, t}$ is estimated as follows

$$
\int_{T_{n, k, r, t}} s_{1}^{-\frac{2 \beta}{2-\alpha_{0}}}\left(t-s_{n}\right)^{-\frac{\alpha+2 \tilde{\alpha}}{2-\alpha_{0}}} \prod_{i=1}^{n-1}\left(s_{i+1}-s_{i}\right)^{-\frac{\alpha}{2-\alpha_{0}}} d s_{1} \cdots d s_{n}
$$




$$
\begin{aligned}
& \leq \int_{0<s_{1}<\cdots<s_{k}<r} s_{1}^{-\frac{2 \beta}{2-\alpha_{0}}}\left(r-s_{k}\right)^{-\frac{\alpha}{2-\alpha_{0}}} \prod_{i=1}^{k-1}\left(s_{i+1}-s_{i}\right)^{-\frac{\alpha}{2-\alpha_{0}}} d s_{1} \cdots d s_{k} \\
& \int_{r<s_{k+1}<\cdots<s_{n}<t} \prod_{i=k+1}^{n-1}\left(s_{i+1}-s_{i}\right)^{-\frac{\alpha}{2-\alpha_{0}}}\left(t-s_{n}\right)^{-\frac{\alpha+2 \tilde{\alpha}}{2-\alpha_{0}}} s_{k+1} \cdots d s_{n} \\
& \leq \frac{c^{n} r^{k\left(1-\frac{\alpha}{2\left(2-\alpha_{0}\right)}+1-\frac{2 \beta}{2\left(2-\alpha_{0}\right)}\right.}}{\Gamma\left(k\left(1-\frac{\alpha}{2\left(2-\alpha_{0}\right)}\right)+1-\frac{2 \beta}{2\left(2-\alpha_{0}\right)}+1\right)} \frac{(t-r)^{(n-k-1)\left(1-\frac{\alpha}{2\left(2-\alpha_{0}\right)}+1-\frac{\alpha+2 \tilde{\alpha}}{2\left(2-\alpha_{0}\right)}\right.} \Gamma\left((n-k-1)\left(1-\frac{\alpha}{2\left(2-\alpha_{0}\right)}\right)+1-\frac{\alpha+2 \tilde{\alpha}}{2\left(2-\alpha_{0}\right)}+1\right)}{\leq(n !)^{\frac{\alpha}{2\left(2-\alpha_{0}\right)}-1} t^{(n-1)\left(1-\frac{\alpha}{2\left(2-\alpha_{0}\right)}\right)+1-\frac{2 \beta}{a\left(2-\alpha_{0}\right)}}(t-r)^{1-\frac{\alpha+2 \tilde{\alpha}}{2\left(2-\alpha_{0}\right)}} .}
\end{aligned}
$$

Thus we have the following bound

$$
B_{n} \lesssim c^{n}(n !)^{\frac{\alpha}{2}-1}|x-y|^{2 \bar{\alpha}}|t-r|^{\frac{4-2 \alpha_{0}-\alpha-2 \tilde{\alpha}}{2}} t^{\tilde{\beta}} t^{\frac{4-2 \alpha_{0}-\alpha}{2} n} .
$$

Denote

$$
\alpha_{0}^{\prime}=\frac{4-2 \alpha_{0}-\alpha-2 \tilde{\alpha}}{4} .
$$

It is easy to verify that $2 \alpha_{0}^{\prime}+\tilde{\alpha}=2-\alpha_{0}-\frac{\alpha}{2}$ and

$$
B_{n} \lesssim c^{n}(n !)^{\frac{\alpha}{2}-1}|x-y|^{2 \bar{\alpha}}|t-r|^{2 \tilde{\alpha}_{0}} t^{\tilde{\beta}} t^{\frac{4-2 \alpha_{0}-\alpha}{2} n} .
$$

Step 6. We estimate $B_{n}$ under hypothesis 3.3 (i). First we have

$$
B_{n} \leq c^{n} n !\left(\int_{\Delta_{r, t}^{n}}\left|\psi_{1}(s)\right|^{\frac{1}{2-\alpha_{0}}} d s\right)^{2-\alpha_{0}},
$$

where

$$
\begin{aligned}
& \psi_{1}(s) \leq \frac{c^{n}}{(n !)^{2}}|x-y|^{2 \tilde{\alpha}} s_{\sigma(1)}^{-\beta} \int_{\mathbb{R}^{(n+1} d}\left|\eta_{n}-\zeta\right|^{2 \tilde{\alpha}} e^{-\left(t-s_{\sigma(n)}\right)\left|\eta_{n}-\zeta\right|^{2}}\left|g_{n}(s, \eta, \zeta)\right| \mu\left(d \xi_{1}\right) \cdots \mu\left(d \xi_{n}\right)\left|\hat{u}_{0}(\zeta)\right| e^{-\frac{s_{\sigma(1)}|\zeta|^{2}}{2}} d \zeta \\
& \leq \frac{c^{n}}{(n !)^{2}}|x-y|^{2 \tilde{\alpha}} s_{\sigma(1)}^{-2 \beta} \int_{\mathbb{R}^{(n+1} d}\left|\eta_{n}-\zeta\right|^{2 \tilde{\alpha}} e^{-\left(t-s_{\sigma(n)}\right)\left|\eta_{n}-\zeta\right|^{2}} \prod_{i=1}^{n-1} e^{-\left(s_{\sigma(i+1)}-s_{\sigma(i)}\right)\left|\eta_{i}-\zeta\right|^{2}} \\
& \prod_{i=1}^{n}\left|\xi_{i}\right|^{\alpha} d \xi_{1} \cdots d \xi_{n} \mid \hat{u}_{0}(\zeta) e^{-\frac{s_{\sigma(1)}|\zeta|^{2}}{2}} d \zeta \\
& \leq \frac{c^{n}}{(n !)^{2}}|x-y|^{2 \tilde{\alpha}} s_{\sigma(1)}^{-2 \beta} \int_{\mathbb{R}^{(n+1} d}\left|\eta_{n}-\zeta\right|^{2 \tilde{\alpha}} e^{-\left(t-s_{\sigma(n)}\right)\left|\eta_{n}-\zeta\right|^{2}} \prod_{i=1}^{n-1} e^{-\left(s_{\sigma(i+1)}-s_{\sigma(i)}\right)\left|\eta_{i}-\zeta\right|^{2}} \\
& \leq \frac{c^{n}}{(n !)^{2}}|x-y|^{2 \tilde{\alpha}} s_{\sigma(1)}^{-2 \beta} \int_{\mathbb{R}^{(n+1} d}\left(\left|\eta_{n}\right|^{2 \tilde{\alpha}}+|\zeta|^{2 \tilde{\alpha}}\right) e^{-\left(t-s_{\sigma(n)}\right)\left|\eta_{n}-\zeta\right|^{2}} \prod_{i=1}^{n-1} e^{-\left(s_{\sigma(i+1)}-s_{\sigma(i)}\right)\left|\eta_{i}-\zeta\right|^{2}} \\
& \quad\left(\left|\eta_{1}\right|^{\alpha}+|\zeta|^{\alpha}\right) \prod_{2=1}^{n}\left(\left|\eta_{i}\right|^{\alpha}+\left|\eta_{i-1}\right|^{\alpha}\right)\left|d \eta_{1} \cdots d \eta_{n}\right| \hat{u}_{0}(\zeta) e^{-\frac{s_{\sigma(1)}|\zeta|^{2}}{2}} d \zeta .
\end{aligned}
$$

In the same way as for (4.13) and (4.14), we have

$$
B_{n} \lesssim c^{n}(n !)^{\frac{\alpha-1}{2}}|x-y|^{2 \bar{\alpha}}|t-r|^{\frac{3-2 \alpha_{0}-2 \tilde{\alpha}-\alpha}{2}} t^{\tilde{\beta}} t^{\frac{3-2 \alpha_{0}-\alpha}{2} n} .
$$

Denote $\tilde{\alpha}_{0}=\frac{3-2 \alpha_{0}-2 \tilde{\alpha}-\alpha}{4}$, then we see easily that $2 \tilde{\alpha}_{0}+\tilde{\alpha}<\frac{3-2 \alpha_{0}-\alpha}{2}$ and

$$
B_{n} \lesssim c^{n}(n !)^{\frac{\alpha-1}{2}}|x-y|^{2 \bar{\alpha}}|t-r|^{2 \tilde{\alpha}_{0}} t^{\tilde{\beta}} t^{\frac{3-2 \alpha_{0}-\alpha}{2} n} .
$$

This proves the proposition.

Theorem 4.3. Assuming that hypothesis 3.2 holds and $u_{0}$ satisfies (3.5). 
(i) Let the hypothesis 3.3 (i) be satisfied and let $\bar{\alpha}_{0}$ and $\bar{\alpha}$ be in $[0,1]$ such that

$$
2 \bar{\alpha}_{0}+\bar{\alpha}<2-\alpha_{0}-\frac{\alpha}{2} .
$$

[It is easy to see the right hand side is positive under our assumption.] Then there is a positive constant $c$ such that for every $t \geq r \geq 0$ and $x, y \in \mathbb{R}^{d}$

$$
\mathbb{E}|u(t, y)-u(r, y)-u(t, x)+u(r, x)|^{p} \leq C \exp \left(c p^{\frac{4-\alpha}{2-\alpha}} t^{\frac{4-2 \alpha_{0}-\alpha}{2-\alpha}}\right)|t-r|^{p \bar{\alpha}_{0}}|x-y|^{p \bar{\alpha}} .
$$

(ii) Let the hypothesis 3.3 (ii) be satisfied and let $\bar{\alpha}_{0}$ and $\bar{\alpha}$ be in $[0,1]$ such that

$$
2 \bar{\alpha}_{0}+\bar{\alpha}<\frac{3-2 \alpha_{0}-\alpha}{2} .
$$

Then there is a positive constant $c$ such that for every $t \geq r \geq 0$ and $x, y \in \mathbb{R}^{d}$

$$
\mathbb{E}|u(t, y)-u(r, y)-u(t, x)+u(r, x)|^{p} \leq C \exp \left(c p^{\frac{3-\alpha}{1-\alpha}} t^{\frac{3-2 \alpha_{0}-\alpha}{1-\alpha}}\right)|t-r|^{p \bar{\alpha}_{0}}|x-y|^{p \bar{\alpha}} .
$$

The above theorem is about the joint Hölder continuity. For the usual Hölder continuity the argument is similar and simpler. We state it as follows.

Theorem 4.4. Assuming that the hypothesis 3.2 and $u_{0}$ satisfies (3.5).

(i) Let the hypothesis 3.3 (i) be satisfied and let $\bar{\alpha}_{0}$ and $\bar{\alpha}$ be in $[0,1]$ such that

$$
\max \left(2 \bar{\alpha}_{0}, \bar{\alpha}\right)<2-\alpha_{0}-\frac{\alpha}{2} .
$$

Then there is a positive constant $c$ such that for every $t \geq r \geq 0$ and $x, y \in \mathbb{R}^{d}$

$$
\mathbb{E}|u(t, x)-u(r, x)|+|u(t, x)-u(t, y)|^{p} \leq C \exp \left(c p^{\frac{4-\alpha}{2-\alpha}} t^{\frac{4-2 \alpha_{0}-\alpha}{2-\alpha}}\right)\left[|t-r|^{p \bar{\alpha}_{0}}+|x-y|^{p \bar{\alpha}}\right] .
$$

(ii) Let the hypothesis 3.3 (ii) be satisfied and let $\bar{\alpha}_{0}$ and $\bar{\alpha}$ be in $[0,1]$ such that

$$
\max \left(2 \bar{\alpha}_{0}, \bar{\alpha}\right)<\frac{3-2 \alpha_{0}-\alpha}{2} .
$$

Then there is a positive constant $c$ such that for every $t \geq r \geq 0$ and $x, y \in \mathbb{R}^{d}$

$$
\mathbb{E}|u(t, x)-u(r, x)|+\left.|u(t, x)-u(t, y)|\right|^{p} \leq C \exp \left(c p^{\frac{3-\alpha}{1-\alpha}} t^{\frac{3-2 \alpha_{0}-\alpha}{1-\alpha}}\right)|t-r|^{p \bar{\alpha}_{0}}|x-y|^{p \bar{\alpha}} .
$$

This theorem combined with the Theorem 2.3 of [5] gives

Corollary 4.5. Assuming that the hypothesis 3.2 and $u_{0}$ satisfies (3.5). Let $\bar{\alpha}_{0}$ and $\bar{\alpha}$ be in $[0,1]$ such that

$$
\max \left(2 \bar{\alpha}_{0}, \bar{\alpha}\right)< \begin{cases}2-\alpha_{0}-\frac{\alpha}{2} & \text { if hypothesis } 3.3 \text { (i) be satisfied } \\ \frac{3-2 \alpha_{0}-\alpha}{2} & \text { if hypothesis } 3.3 \text { (ii) be satisfied }\end{cases}
$$

(i) For any $M>0$ there is a random constant $C_{M}$ such that every $t, r \in[0, M]$ and for every $x, y \in \mathbb{R}^{d}$ satisfying $|x|,|y| \leq M$,

$$
|u(t, y)-u(r, y)-u(t, x)+u(r, x)| \leq C|t-r|^{\bar{\alpha}_{0}}|x-y|^{\bar{\alpha}} .
$$

(ii) For any $M>0$ there is a random constant $C_{M}$ such that every $t, r \in[0, M]$ and for every $x, y \in \mathbb{R}^{d}$ satisfying $|x|,|y| \leq M$,

$$
|u(t, x)-u(r, x)|+|u(t, y)-u(t, x)| \leq V\left[|t-s|^{\bar{\alpha}_{0}}+|x-y|^{\bar{\alpha}}\right] .
$$

Remark 4.6. When $d=1$ and $W$ is a space-time white noise, this corresponds to the case $\alpha_{0}=\alpha=1$ ans we can use the result for the case when the hypothesis 3.3 (i) is satisfied. The above corollary says if $2 \bar{\alpha}_{0}+\bar{\alpha}<1 / 2$, then

$$
|u(t, y)-u(r, y)-u(t, x)+u(r, x)| \leq C|t-r|^{\bar{\alpha}_{0}}|x-y|^{\bar{\alpha}} .
$$

This coincides with the optimal Hölder exponent result in [5]. On the other hand, the corollary also implies in this case that if $\bar{\alpha}_{0}<1 / 4$ and $\bar{\alpha}<1 / 2$, then

$$
|u(t, x)-u(r, x)|+|u(t, y)-u(t, x)| \leq C\left[|t-r|^{\bar{\alpha}_{0}}+|x-y|^{\bar{\alpha}}\right] .
$$


From here we see that $u(t, x)$ is Hölder continuous in $t$ with exponent $\bar{\alpha}_{0}<1 / 4$ and is Hölder continuous in $x$ with exponent $\bar{\alpha}<1 / 2$. This is the optimal Hölder modulus of continuity for the solution of (1.1) with one dimensional space-time white noise.

\section{REFERENCES}

[1] Balan, R.; Quer-Sardanyons, L. and Song, J. Hölder continuity for the Parabolic Anderson Model with space-time homogeneous Gaussian noise. arXiv preprint.

[2] Chen, L. and Dalang, R. C. Hölder-continuity for the nonlinear stochastic heat equation with rough initial conditions. Stoch. Partial Differ. Equ. Anal. Comput. 2 (2014), no. 3, 316-352.

[3] Chen, L.; Kalbasi, K.; Hu, Y. and Nualart, D. Intermittency for the stochastic heat equation driven by time-fractional Gaussian noise with $H \in(0,1 / 2)$. To appear in Prob. Theory and Related Fields.

[4] Hu, Y. Analysis on Gaussian space. World Scientific, Singapore, 2017.

[5] Hu, Y. and Le, . A multiparameter Garsia-Rodemich-Rumsey inequality and some applications. Stochastic Process. Appl. 123 (2013), no. 9, 3359-3377.

[6] Hu, Y., Nualart, D. Stochastic heat equation driven by fractional noise and local time. Probab. Theory Related Fields 143 (2009), no. 1-2, 285-328.

[7] Hu, Y., Huang, J., Lê, K., Nualart, D. and Tindel, S. Stochastic heat equation with rough dependence in space. Ann. Probab. 45 (2017), no. 6B, 4561-4616.

[8] Hu, Y., Huang, J., Lê, K., Nualart, D. and Tindel, S. Parabolic Anderson model with rough dependence in space. To appear.

[9] Hu, Y., Huang, J., Nualart, D., Tindel, S. Stochastic heat equations with general multiplicative Gaussian noises: Hölder continuity and intermittency. Electron. J. Probab. 20 (2015), no. 55, 50 pp.

[10] Hu, Y.; Nualart, D. and Song, J. A nonlinear stochastic heat equation: Hölder continuity and smoothness of the density of the solution. Stochastic Process. Appl. 123 (2013), no. 3, 1083-1103.

[11] Hu, Y., Nualart, D., Song, J. Feynman-Kac formula for heat equation driven by fractional white noise. Ann. Probab. 30, 291-326.

[12] Memin, J.; Mishura Y. and Valkeila, E. Inequalities for the moments of Wiener integrals with respect to a fractional Brownian motion. Statist. Probab. Lett. 51 (2001) 197-206.

[13] Nualart, D. The Malliavin calculus and related topics. Second edition. Probability and its Applications (New York). Springer-Verlag, Berlin, 2006. xiv+382 pp.

[14] Sanz-Solé, M. and Sarrà, M. Hölder continuity for the stochastic heat equation with spatially correlated noise. Seminar on Stochastic Analysis, Random Fields and Applications, III (Ascona, 1999), 259-268, Progr. Probab., 52, Birkhäuser, Basel, 2002.

Department of Mathematical and Statistical Sciences, University of Alberta at Edmonton, Edmonton, AlBERTA, T6G 2G1, CANADA

E-mail address: yaozhong@ualberta.ca

Department of Mathematics, South Kensington Campus, Imperial College London, London, SW7 2AZ, United KINGDOM

E-mail address: n.le@imperial.ac.uk 